\title{
Personalization of learning activities within a virtual envi- ronment for training based on fuzzy logic theory
}

\author{
Fahim Mohamed*, Jakimi Abdeslam, El Bermi Lahcen \\ Software Engineering \& Information Systems Engineering Team UMI, Faculty of Sciences and Technology, Errachidia, Morocco.
}

\begin{abstract}
The development of computers and multimedia technology has opened up new possibilities for training based on virtual reality. Virtual reality is the most powerful extension of simulation based systems. In virtual reality there is a move to three dimensional, multi-sensory interfaces. A virtual environment for training (VET) can be defined as a computer-generated environment based on virtual reality, to simulate the real world. Learning through a VET can personalize learning needs for learners to promote the quality of learning. However, learners can't be provided with appropriate learning activities because often there is no personalized service to respond to each learner's particular needs. The obvious solution is to generate learning activities based on each learner's profile. Yet it is a complex process, especially with the inaccuracy of data that may contains a learner's profile. The main goal of this paper is to associate suitable learning activities to each learner based on his profile, to do so, we propose to employ fuzzy logic technique, and the fuzzy inference system to handle reasoning under uncertainty and inaccuracy which is one major issue of great concern in learner model design.
\end{abstract}

Keywords: Virtual environments for training, learning activities, fuzzy logic, fuzzy inference system.

2010 MSC: 68T05, 68T27.

(C) 2018 All rights reserved.

\section{Introduction}

Based on virtual reality [4], VETs offer numerous benefits in training applications. First, they allow extensive user interactions in a very convenient and natural manner. Second, VETs have proven to be advantageous to put learners into varied training situations to acquire knowledge and competencies, especially when these situations are taking place in uncontrolled circumstances, or when they are dangerous, unrealizable, or expensive to establish in reality. Learning in an unconfined environments like a VET, demands even more personalization approaches to provide learners with personalized learning activities to assure the quality and the effectiveness of learning.

In our context, a learning activity contains several features of information necessary to achieve the goal of training, such as content description, succession of actions to be done, prerequisite information

\footnotetext{
*Corresponding author

Email addresses: fahim.mohamed89@gmail.com (Fahim Mohamed), ajakimi@yahoo.fr (Jakimi Abdeslam), elbermi.lahcen@gmail.com (El Bermi Lahcen)
}

doi: $10.22436 / \mathrm{mns} .03 .01 .02$

Received: 2017-06-03 Revised: 2018-06-01 Accepted: 2018-06-10 
and so on. Learning activities personalization (LAP) can be defined as the process of tailoring the content and structure learning activities to the specific and individual needs of each learner after accessing his personal requirements. LAP is one of the most important ways of improving the effectiveness of learning. At the same time, it is also a complex process that requires consideration of numerous factors such as learner 's profiles. LAP becomes an issue with the uncertainty and imprecision of data that may contains a learner profile. In this paper an attempt to integrate the fuzzy logic theory into the process of the personalization of learning activities is presented. In the following, we first present the related works to the personalization of learning activities. Then, we present our methodology, finally, we conclude and talk about our future works.

\section{Related work}

The objective of this section is to present research's work related to personalization of learning. The design and development of an adaptive learning system can be challenging and a complex task due to the individual differences that exist among learners. Every learner has his peculiar needs, requirements, preferences and characteristics. Recently, many works related to the personalization of learning have been developed with the aim of improving the adaptatively in educational systems. In our work, the goal is to associate suitable learning activities, pedagogical resources, etc. to each learner based on his profile. A profile contains information such as: learner 's knowledge level, desired difficulty level, age, etc.. To allow this personalization of learning, many solutions have been proposed. Current methods for personalization of learning can be divided into three groups: (i) oriented activities approaches [14]: where the learning process is represented by a graph in which the activities are identified and decomposed; (ii) oriented resources approaches [1, 6, 19]: where the learning process returns to select, assemble and present contents, (iii) oriented objectives approaches $[2,18]$ : where the learning process is seen as a process of satisfaction of pedagogical objectives already defined. These approaches use a set of algorithms and techniques from artificial intelligence and web semantics such as ant colony optimization $[7,8,17,20]$, Bayesian networks [2], the algorithm of support vector machines (SVM) [15], ontologies [5, 16], web services technology [11], etc.. However, these methods are quite limited in term of handling uncertain and imprecise data.

\section{Fuzzy logic theory}

Fuzzy logic has rapidly become one of the most effective and successful of today's technologies for developing control applications. Fuzzy logic addresses such applications perfectly as it emulates human decision making with an ability to generate precise solutions from uncertain and inaccurate data. It is an approach to computing based on "membership degree" rather than the usual "true or false" Boolean logic. Fuzzy logic seems closer to the way our brains work. For instance, to answer a question about a hotel services, most time answers could be "Not Very Satisfied" or "Quite Satisfied", which are also fuzzy or ambiguous answers. Exactly to what degree is one satisfied or dissatisfied with some hotel services? These vague answers can only be created and implemented by human beings, but not machines. So, how can computers and machines handle those vague data? Based on this observation, Zadeh [21, 22] developed fuzzy set theory that generalizes classical set theory (Boolean logic) to allow the notion of partial membership. This invention was not well recognized until Dr. E. H. Mamdani, who is a professor at London University, applied the fuzzy logic in a practical application to control an automatic steam engine in 1974 [12]. The use of fuzzy logic allows working with quantitative and qualitative descriptions. In fuzzy set theory, an element can belong entirely to a set (degree of belonging is 1), or "almost" belong to it (with a degree of belonging equal to 0.9 for example). Fuzzy logic has been successfully employed in a variety of applications in recent years $[9,13,20]$.

An example of a fuzzy set could be " the set of temperature", it can take a fuzzy value (e.g., cold, cool, tepid, warm, and hot). Each fuzzy value such as hot is called a linguistic variable. A fuzzy set can be 
represented by a membership function. This function indicates the grade (degree) of membership within the set, of any element of the universe of discourse. The membership function maps every element of the universe to numerical values in the interval $[0,1]$. Specifically, let consider, $\mu_{\mathrm{A}}$ the membership function of the set A, $\mathrm{U}$ a universe of discourse, in the classical set theory:

$$
\forall x \in U, \mu_{A}(x)=0 \text { if } x \notin A, \quad \mu_{A}(x)=1 \text { if } x \in A .
$$

In the context of fuzzy set theory, a fuzzy set $\mathrm{A}$ of $\mathrm{U}$ is characterized by a membership function $\mu_{\mathrm{A}}$ defined by:

$$
\mu_{\mathrm{A}}: \mathrm{U} \rightarrow[0.1], \quad x \rightarrow \mu_{\mathrm{A}}(x) .
$$

$\mu_{A}$ associates to each object $x$ of $U$ a value in the interval $[0,1]$ which represents the degree of membership of $x$ to A.

A membership function value of zero indicates that corresponding element is definitely not an element of the fuzzy set. A membership function value of one implies that the corresponding element is definitely an element of the fuzzy set. A grade of membership greater than 0 and less than 1 corresponds to a non-crisp (or fuzzy) membership, and the corresponding elements fall on the fuzzy boundary of the set. There is many applications of fuzzy logic and fuzzy sets, such as fuzzy inference systems (FIS), fuzzy decision trees, etc.. We have chosen FIS, because it seems to be the most suitable for our approach of personalization of learning activities.

\section{Architecture of the fuzzy proposed approach}

In this paper we intend to employ the use of fuzzy logic to model the learner's profile and fuzzy inference system to classify learners with the aim of improving the quality of learning and increase the systems adaptability. The use of fuzzy logic in this context is to enable the computational analysis of the learner's characteristics and in order to handle the uncertainty issues related to the learner model design. The architecture of the system we are developing is shown in the Figure 1. The first step in the operation of our system is to collect user's data to build a learner's profile from information given by each user through user interface. Information which forms a learning profile are the following.

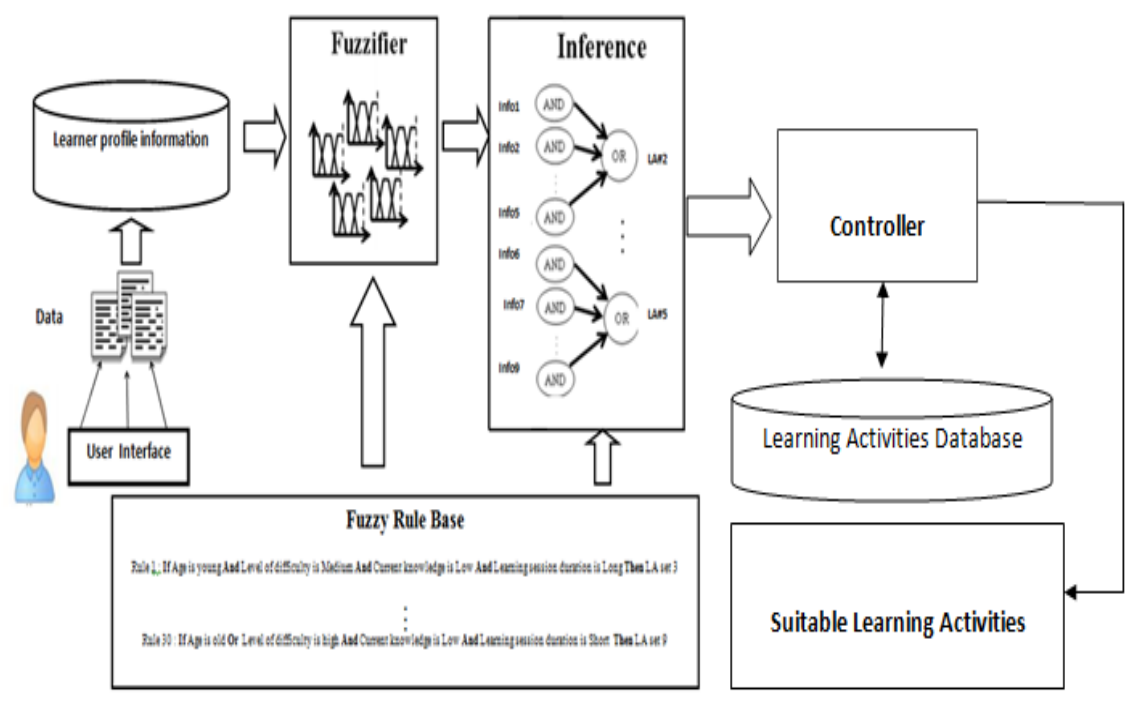

Figure 1: Overview of our approach.

- learning session duration; 
- gender;

- level of difficulty;

- desired language;

- current knowledge level;

- age.

The second step is fuzzification. The fuzzifier converts the input variables also known as the crisp inputs to a linguistic variables or fuzzy terms using the membership functions stored in the fuzzy rule base. Membership functions can have different shapes. The most commonly used membership functions in fuzzification processes are trapezoidal, triangular, Gaussian, and Sigmoidal membership functions. In our case we used the triangular. For example, for the fuzzification of the learning session duration $x=70$ min, the two membership functions $\mu_{A}$ and $\mu_{B}$ from Figure 2 can be used, which characterize a low and a medium Learning session duration fuzzy set, respectively. The given duration value of $x=70 \mathrm{~min}$ belongs with a grade of $\mu_{A} x=0.75$ to the fuzzy set "low" and with a grade of $\mu_{B} x=0.25$ to the fuzzy set "medium".

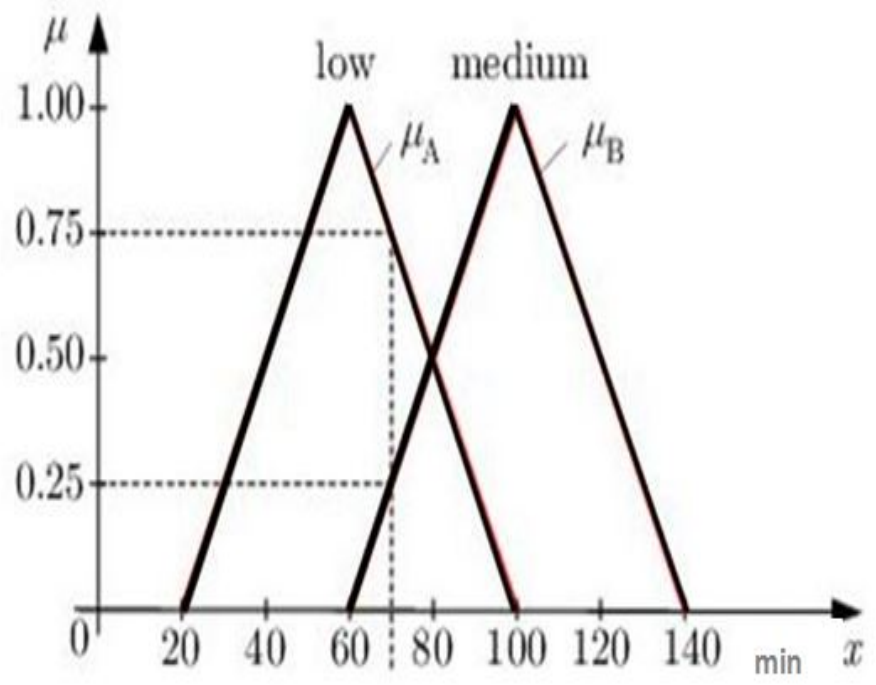

Figure 2: Fuzzification of a learning session duration.

Afterwards, the inference engine which is the kernel of decision making process in a fuzzy inference system use the fuzzy rule base to make deductive reasoning which in turn allow the system to infer conclusions. The fuzzy rule base composed of expert IF $\ll$ antecedents $\gg$ THEN $\ll$ conclusions $\gg$ rules. These rules transform the input variables (the learner profile information) to an output that will tell us the suitable learning activities.

The following rule is an example of a fuzzy If-Then rule.

If (age is young) and (level of difficulty is medium ) and (current knowledge is low) and (learning session duration is long), then learning activities are the "LA set 3". Table 1 shows more examples of fuzzy rules. For information "gender" and "desired language" are not taken into consideration during the inference phase. They will be considered automatically on the execution phase. Finally, the controller, based on the value given by the inference engine, picks up from the "learning activities database", the suitable learning activities set appropriate to the learner profile of the input. 
Table 1: Fuzzy rules examples.

\begin{tabular}{l}
\hline FuzZy rules \\
\hline Rule 1: If ( Age is Young) And (Level of difficulty is Low ) And (Current knowledge is High) And (Learning session duration is Short) Then "LA 6" \\
Rule 2: If (Age is Old) or (Level of difficulty is Low) And (Current knowledge is Low) And (Learning session duration is Long) Then 'LA 4 \\
Rule 3: If (Age is Adult) Or (Level of difficulty is High) And (Current knowledge is Medium) And (Learning session duration is Long) Then "LA 7" \\
Rule 4: If (Age is Young) Or (Level of difficulty is Low) And (Current knowledge is Medium) And (Learning session duration is Long) Then "LA 5" \\
Rule 5: If (Age is Adult) And (Level of difficulty is High) And (Current knowledge is High) And (Learning session duration is Medium) Then "LA 8" \\
Rule 6: If (Age is Old) Or (Level of difficulty is Medium) And (Current knowledge is High) And (Learning session duration is Medium) Then "LA 10" \\
Rule 7: If (Age is Adult) And (Level of difficulty is High) Or (Current knowledge is High) And (Learning session duration is Long) Then "LA 12" \\
Rule 8:If (Age is Young) And (Level of difficulty is Low) And (Current knowledge is Low) And (Learning session duration is Long) Then "LA 17" \\
Rule 9: If (Age is Young) Or (Level of difficulty is Medium) And (Current knowledge is Medium) Or (Learning session duration is Medium) Then "LA 14" \\
Rule 10: If (Age is Adult) And (Level of difficulty is Medium) And (Current knowledge is Medium) And (Learning session duration is Medium) Then "LA 11
\end{tabular}

\section{Conclusion}

This paper focuses on the importance of using fuzzy logic in learner modeling and decision making process within a VET. The main advantage of this proposed approach is that it is efficient in handling the uncertainty in the learner's profiles. The fuzzy inference engine uses the rule base for accurately categorizing the learners even with incomplete information. Based on the value given by the fuzzy inference engine, the controller generate suitable learning activities for each learner. However, a number of further data, in particular, learner's feedback information is required to promote the functionality of the system. The ongoing work aims at including this proposed approach in our previous work [3], in order to generate adaptable and effective pedagogical scenarios for VET.

\section{References}

[1] L. de Marcos, J.-J. Martinez, J.-A. Gutierrez, Swarm intelligence in e-learning: a learning object sequencing agent based on competencies, The 10th annual conference on Genetic and evolutionary computation, 2008 (2008), 17-24. 2

[2] J. El Bouhdidi, M. Ghailani, A. Fennan, An intelligent architecture for generating evolutionary personalized learning paths based on learner profiles, J. Theoretical Appl. Infor. Tech. (JATIT), 57 (2013), 294-304. 2

[3] M. Fahim, A. Jakimi, L. El Bermi, Pedagogical Scenarization for Virtual Environments for Training: Towards Genericity, Coherence and Adaptivity, Int. J. Adv. Eng. Res. Sci. (IJAERS), 12 (2016), 96-103. 5

[4] P. Fuchs, G. Moreau, Le traité de la réalité virtuelle, Les Presses de l'Ecole des Mines de Paris, Paris, (2006). 1

[5] M. Ghailani, J. E. Bouhdidi, A. Fennan, Towards an adaptive e-learning solution based on ontologies and competencies approach, Int. J. Comput. Appl., 98 (2014), 42-52. 2

[6] P. Karampiperis, D. Sampson, Automatic learning object selection and sequencing in web-based intelligent learning systems, Idea Group Inc., 2006 (2006), 56-71. 2

[7] A. A. Kardan, M. A. Ebrahim, M. B. Imani, A new personalized learning path generation method: Acomap, Indian J. Sci. Res., 5 (2014), 17-24. 2

[8] A. Kumar, J. E. Nalavade, V. Yeola, V. Vivek, Y. Srivastava, An adaptive learning system based on ant colony algorithm, Int. J. Soft Comput. Eng. (IJSCE), 3 (2013), 1-3. 2

[9] C. T. Lin, C. L. Chin, Using fuzzy inference and cubic curve to detect and compensate backlight image, Int. J. Fuzzy Syst., 8 (2006), 2-13. 3

[10] C. T. Lin, K. W. Fan, C. M. Yeh, H. C. Pu, F. Y. Wu, High-accuracy skew estimation of document images, Int. J. Fuzzy Syst., 8 (2006), 119-126.

[11] F. Lin, P. Holt, S. Leung, Q. Li, A multiagent and service-oriented architecture for developing adaptive e-learning systems, Int. J. Cont. Eng. Edu. Life Long Learning, 16 (2006), 77-91. 2

[12] E. H. Mamdani, S. Assilion, An Experiment in Linguistic Synthesis With a Fuzzy Logic Controller, Int. J. Man-Machine Stud., 7 (1975), 1-13. 3

[13] N. Marion, Modélisation de scénarios pédagogiques pour les environnements de réalité virtuelle d'apprentissage humain, PhD Thesis (Université de Bretagne Occidentale), France, (2010). 3

[14] A. Naji, M. Ramdani, Using the ant colony algorithm to establish the best path of learning activities, Appl. Math. Sci., 7 (2013), 3873-3881. 2 
[15] E.-A. Ouraiba, A. Chikh, A. Taleb-Ahmed, Z. El Yeb dri, Automatic personalization of learning scenarios using svm, 2009 Ninth IEEE International Conference on Advanced Learning Technologies, 2009 (2009), 183-185. 2

[16] Q. D. Pham, A. M. Florea, Adaptation To Learning Styles In A Multi-Agent ELearning System, Internet Learning, 2 (2013), 10 pages. 2

[17] M. Pushpa, ACO in e-learning: Towards an adaptive learning path, Int. J. Comput. Sci. Eng. (IJCSE), 4 (2012), 11 pages. 2

[18] S. Talhi, M. Djoudi, S. Ouadfel, S. Zidat, Un canevas de tuteur intelligent hypermédia pour l'apprentissage á distance universitaire, Proc. 4th International Conference on Computer Integrated Manufacturing, 2007 (2007), 5 pages. 2

[19] G. Valigiani, E. Lutton, C. Fonlupt, P. Collet, Optimisation par "hommiliére" de chemins pédagogiques pour un logiciel d'e-learning, Technique et Science Informatiques, 26 (2007), 1245-1268. 2

[20] M. Wallace, S. Ioannou, K. Karpouzis, S. Kollias, Possibilistic rule evaluation: A case study in facial expression analysis, Int. J. Fuzzy Syst., 8 (2006), 219-223. 2, 3

[21] L. A. Zadeh, Fuzzy set, Information and Control, 8 (1965), 338-353. 3

[22] L. A. Zadeh, The concept of a linguistic variable and its application to approximate reasoning-I, Information Sci., 8 (1975), 199-249. 3 